Gut and Liver, Vol. 11, No. 2, March 2017, pp. 290-297

\title{
Diagnostic Value of Endoscopic Ultrasonography in Symptomatic Patients with High and Intermediate Probabilities of Common Bile Duct Stones and a Negative Computed Tomography Scan
}

\author{
Tae Joo Jeon ${ }^{1,2}$, Jae Hee Cho ${ }^{3}$, Yeon Suk Kim ${ }^{3}$, Si Young Song ${ }^{2,4}$, and Ji Young Park ${ }^{1}$ \\ ${ }^{1}$ Division of Gastroenterology, Department of Internal Medicine, Inje University Sanggye Paik Hospital, Inje University College of Medicine, \\ ${ }^{2}$ Department of Internal Medicine, The Graduate School, Yonsei University College of Medicine, Seoul, ${ }^{3}$ Division of Gastroenterology, Department \\ of Internal Medicine, Gachon University Gil Medical Center, Incheon, and ${ }^{4}$ Brain Korea 21 Project for Medical Science, Yonsei University College \\ of Medicine, Seoul, Korea
}

Background/Aims: When computed tomography (CT) does not indicate choledocholithiasis in highly suspicious patients, there is no definite consensus on the subsequent modality. Endoscopic ultrasonography (EUS) indicates fewer procedurerelated complications than endoscopic retrograde cholangiopancreatography (ERCP) and has a lower cost than magnetic resonance cholangiopancreatography. Therefore, we aimed to investigate the diagnostic value of EUS in patients with suspected choledocholithiasis and negative CT findings. Methods: Between March 2008 and November 2014, we retrospectively evaluated 200 patients with negative CT findings and high or intermediate probabilities of choledocholithiasis. All patients initially underwent EUS followed by ERCP as a confirmatory criterion standard. The primary outcome in these patients was the accuracy of EUS in the detection of choledocholithiasis. The secondary outcome was the clinical prediction of common bile duct (CBD) stones in this group. Results: EUS indicated choledocholithiasis in 165 of the 200 patients, and ERCP confirmed choledocholithiasis in 161 patients (80.5\%). The accuracy of EUS in the detection of choledocholithiasis was $94.0 \%$ (sensitivity, 97.5\%; specificity, 79.5\%; positive predictive value, 95.2\%; negative predictive value, $88.6 \%$ ). A multivariate analysis demonstrated that choledocholithiasis was strongly predicted by EUS detection of choledocholithiasis, an age $>55$ years and a clinical diagnosis of cholangitis. Conclusions: An EUS-first approach is recommended for patients with suspected CBD stones and negative CT findings. (Gut Liver 2017;11:290-297)

Key Words: Choledocholithiasis; Endosonography; Cholangiopancreatography, endoscopic retrograde

\section{INTRODUCTION}

Choledocholithiasis is a complication of gallstone disease that occurs in approximately 10\% of patients with symptomatic cholelithiasis. ${ }^{1,2}$ It is important to diagnose common bile duct (CBD) stones, because they can cause morbidity (e.g., pancreatitis and cholangitis) and mortality, especially if treatment is delayed. ${ }^{2,3}$ The initial evaluation of suspected choledocholithiasis should include biochemical liver function tests, such as the levels of alkaline phosphatase (ALP), total bilirubin, alanine aminotransferase (ALT), and aspartate aminotransferase (AST), as well as transabdominal ultrasonography of the right upper quadrant. Many investigators have noted that the probability of a CBD stone is increased in the presence of multiple prognostic signs, and so the American Society for Gastrointestinal Endoscopy (ASGE) criteria are used to categorize patients as having a low, intermediate, or high probability of having choledocholithiasis, using their clinical and biochemical findings. ${ }^{4}$ In practice, the majority of patients with choledocholithiasis visit the emergency room, and so computed tomography (CT) is often the firstline diagnostic modality, due to its convenience and its use in the differential diagnosis of acute abdomen (e.g., perforation). ${ }^{1,2}$

Correspondence to: Ji Young Park ${ }^{\mathrm{a}}$ and Jae Hee Cho ${ }^{\mathrm{b}}$

${ }^{a}$ Division of Gastroenterology, Department of Internal Medicine, Inje University Sanggye Paik Hospital, Inje University College of Medicine, 1342 Dongil-ro, Nowon-gu, Seoul 01757, Korea

Tel: +82-2-950-1340, Fax: +82-2-950-1955, E-mail: human@paik.ac.kr

${ }^{b}$ Division of Gastroenterology, Department of Internal Medicine, Gachon University Gil Medical Center, 21 Namdong-daero, 774beon-gil, Namdonggu, Incheon 21565, Korea

Tel: +82-32-460-3213, Fax: +82-32-461-3214, E-mail: jhcho9328@gilhospital.com

Received on January 26, 2016. Revised on May 19, 2016. Accepted on June 30, 2016. Published online December 16, 2016

pISSN 1976-2283 eISSN 2005-1212 https://doi.org/10.5009/gnl16052

() This is an Open Access article distributed under the terms of the Creative Commons Attribution Non-Commercial License (http://creativecommons.org/licenses/by-nc/4.0) which permits unrestricted non-commercial use, distribution, and reproduction in any medium, provided the original work is properly cited. 
If choledocholithiasis is identified on CT, therapeutic endoscopic retrograde cholangiopancreatography (ERCP) is appropriate. ${ }^{1}$ However, diagnostic CT often fails to detect radiolucent choledocholithiasis, such as cholesterol and small pigment stones, despite clinical and biochemical findings that are consistent with choledocholithiasis. ${ }^{2,3,5}$

Endoscopic ultrasonography (EUS) is an excellent method for detecting CBD stones, with a sensitivity and specificity of approximately 95\%. In the last decade, numerous studies have demonstrated that EUS is as accurate as ERCP for detecting CBD stones, and that it can eliminate unnecessary ERCP procedures and ERCP-related complications. ${ }^{3,6-9}$ Recently, the utility of magnetic resonance cholangiopancreatography (MRCP) has also been highlighted. Five randomized, prospective, comparative studies were performed to compare the accuracy of choledocholithiasis detection between EUS and MRCP. All studies showed no significant differences in sensitivity, specificity, positive and negative predictive values and likelihood ratio between two modalities. ${ }^{10-14}$ Meta-analysis of five randomized trials showed that the aggregated sensitivity, specificity, positive and negative values of EUS were 0.93, 0.96, 0.93, and 0.96, respectively; of MRCP they were $0.85,0.93,0.87$, and 0.92, respectively. These aggregated values were not significantly different. ${ }^{15}$ Both modalities had their own merit; most of all, EUS showed greater convenience because, if choledocholithiasis was found, the operator could perform ERCP on the spot. As well, compared with MRCP, EUS showed good performance for small stones and visualization of the biliary tree because it offered high resolution, and multiple images could be reconstructed. EUS could be performed on claustrophobic patients; and the cost of EUS was lower than that of MRCP. However, EUS accuracy was highly dependent upon the operator and the result could have been influenced by the operator's expertise. Even a diagnostic EUS carried the risk of sedation and related complications such as perforation and bleeding. Meanwhile, the role of EUS is not fully established in patients with suspected choledocholithiasis and negative CT findings. ${ }^{1}$ Therefore, we aimed to investigate the usefulness of EUS in patients with an intermediate or high probability of suspected CBD stones and negative CT findings.

\section{MATERIALS AND METHODS}

\section{Patients and study design}

This retrospective study evaluated data (March 2008 to November 2014) from a prospective EUS/ERCP registry maintained by Inje University Sanggye Paik Hospital, Seoul, Korea and Myongji Hospital, Goyang, Korea. From this registry, we identified 200 patients with suspected CBD stones and negative CT findings. All patients underwent EUS to evaluate CBD stones and choledocholithiasis was confirmed by ERCP (Fig. 1). The patients' clinical risk of choledocholithiasis was assessed using the ASGE guidelines, which provide a management algorithm for patients with symptomatic cholelithiasis according to their probability of having choledocholithiasis. Based on these criteria, patients were categorized as having a low $(<10 \%)$, intermediate (10\% to 50\%), or high (>50\%) probability of choledocholithiasis, using their age, liver function test results, and transabdominal ultrasonography findings. ${ }^{4}$ We excluded patients who had been diagnosed with pancreatobiliary cancer, had undergone cholecystectomy, or were $<18$ years old. Gachon University Gil Medical Center Institutional Review Board approved this retrospective study's design (GDIRB2015-53).

\section{EUS/ERCP}

Two endosonographers with at least 1,000 procedures of experience performed the EUS using a radial scanning echoendoscope (GF-UE260-AL5/UM-240; Olympus Corp., Tokyo, Japan). Positive identification of CBD stones was defined as a
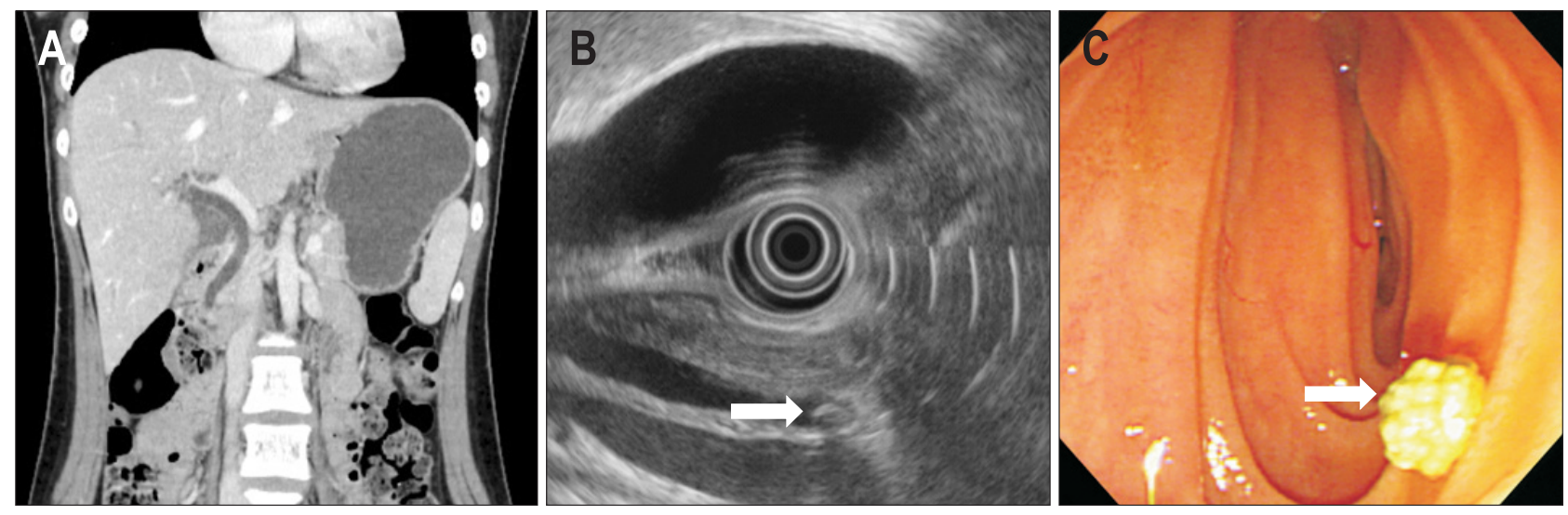

Fig. 1. Common bile duct stone findings on endoscopic ultrasonography (EUS) and endoscopic retrograde cholangiopancreatography (ERCP) in patients with negative computed tomography findings. (A) No choledocholithiasis is apparent on computed tomography in the coronal view. (B) A 4-mm ovoid hyperechoic lesion with posterior acoustic shadowing (white arrow) identified in the distal common bile duct during EUS. (C) A 4-mm cholesterol stone (white arrow) removed by ERCP. 
hyperechoic focus, with or without an acoustic shadow, which was observed within the CBD stone. The size of the CBD stones and the widths of the CBD were measured.

ERCP was performed as a criterion standard for confirmation of choledocholithiasis within 1 day after EUS using a TJF-240/260 duodenoscope (Olympus Corp.) by the same endoscopist who performed the EUS. After selective biliary cannulation, a cholangiogram was obtained, and sphincterotomy was performed when indicated. If a filling defect or stones were present in the $\mathrm{CBD}$, bile duct sweeping and stone extraction were performed using a retrieval balloon and/or basket. When deep biliary cannulation was difficult, an aggressive cannulation technique, such as infundibulotomy, needle-knife sphincterotomy, or transpancreatic papillary septotomy, was used. The stone's size was calculated on cholangiography when possible. A definitive diagnosis of choledocholithiasis was made based on visual assessment after stone extraction.

\section{Definitions and assessment of outcomes}

Biliary colic was defined as pain that was localized to the right upper quadrant or epigastrium. This pain usually started abruptly, persisted without fluctuation, and resolved gradually over 2 to 4 hours. ${ }^{16}$ Clinical pancreatitis was defined as new or worsened abdominal pain that lasted for $>24$ hours with elevated serum amylase and/or lipase levels that were $>3$-fold above the upper normal limits. ${ }^{17}$ Diagnosis of clinical cholangitis was based on the 2013 Tokyo guidelines that were proposed for the diagnosis of acute cholangitis. According to these criteria, cholangitis should be suspected if a patient has fever and/or shaking chills, or laboratory evidence of an inflammatory response (abnormal white blood cell counts, increased serum C-reactive protein levels, or other changes that are suggestive of inflammation) with jaundice or abnormal serum liver biochemistry (elevated ALP, AST, ALT, or $\gamma$-glutamyl transpeptidase levels). ${ }^{16}$ Procedure-related complications, including post-ERCP pancreatitis (PEP), bleeding, and perforation, were graded according to the consensus criteria as mild (2 to 3 days of hospitalization), moderate (4 to 10 days of hospitalization), or severe ( $>10$ days of hospitalization). PEP was diagnosed based on the presence of typical abdominal pain that was associated with elevated serum amylase levels that were $>3$-fold above the upper normal limit at 24 hours after the procedure. Bleeding was defined as clinical evidence of hemorrhage, such as melena or hematemesis, with a $>2 \mathrm{~g} / \mathrm{dL}$ decrease in hemoglobin levels or the need for transfusion. Perforation was assessed during the procedure by visual inspection, or after the procedure by plain radiography.

\section{Statistical analysis}

Categorical variables were analyzed using the chi-square test or Fisher exact test. Continuous variables were presented as mean \pm standard deviation, and were analyzed using the MannWhitney test. Multivariate logistic regression analysis was used to evaluate the predictors of choledocholithiasis. The sensitivity, specificity, positive and negative predictive values, and diagnostic accuracy were all calculated using the 95\% confidence interval (CI). A p-value of $<0.05$ was chosen as the significance threshold for all tests, given the Bonferroni correction that was conducted among the study cohort. All statistical analyses were performed using SPSS software version 18.0 (SPSS Inc., Chicago, IL, USA).

\section{RESULTS}

A search of our database identified 200 patients with negative CT findings and an intermediate or high clinical probability of having CBD stones. Among these 200 patients, EUS revealed CBD stones in 165 patients (82.5\%), and 161 patients (80.5\%) were confirmed to have CBD stones via ERCP (Fig. 2). Eight patients (4.9\%) were ultimately found not to have CBD stones using ERCP, despite positive EUS findings. In contrast, four patients (11.4\%) with negative EUS findings and suspicious clinical symptoms were confirmed to have CBD stones using ERCP. The choledocholithiasis detection rate in patients categorized with high and intermediate probability of choledocholithiasis according to ASGE guidelines is shown in Figs 3 and 4. Sixty patients (83.3\%) with high probability had CBD stones among

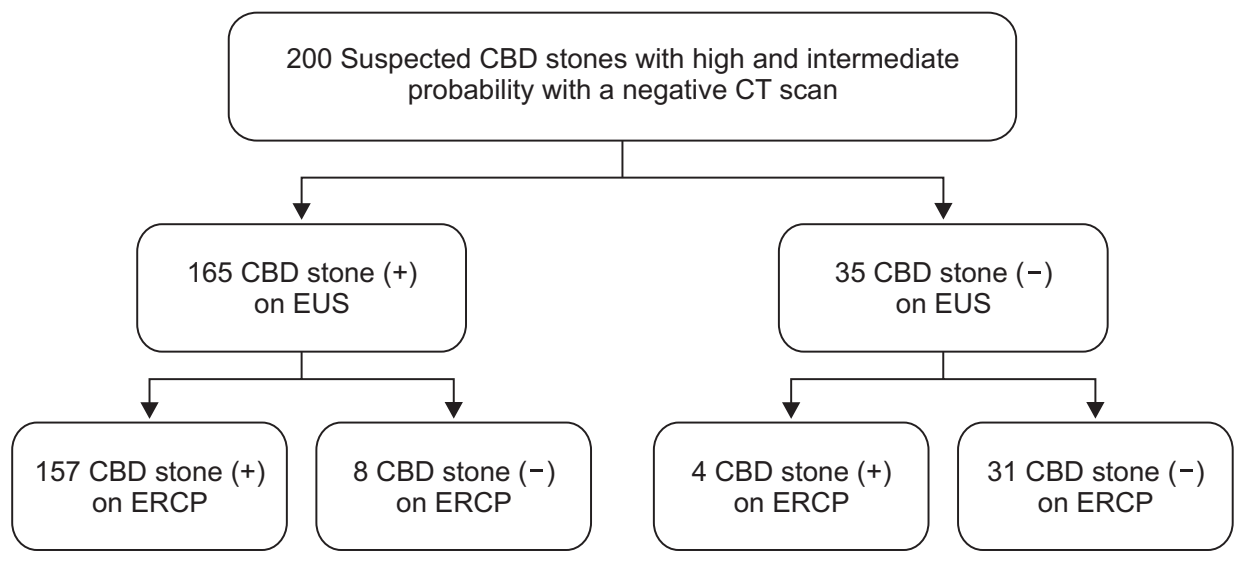

Fig. 2. Study flow chart. CBD, common bile duct; $\mathrm{CT}$, computed tomography; EUS, endoscopic ultrasonography; ERCP, endoscopic retrograde cholangiopancreatography. 

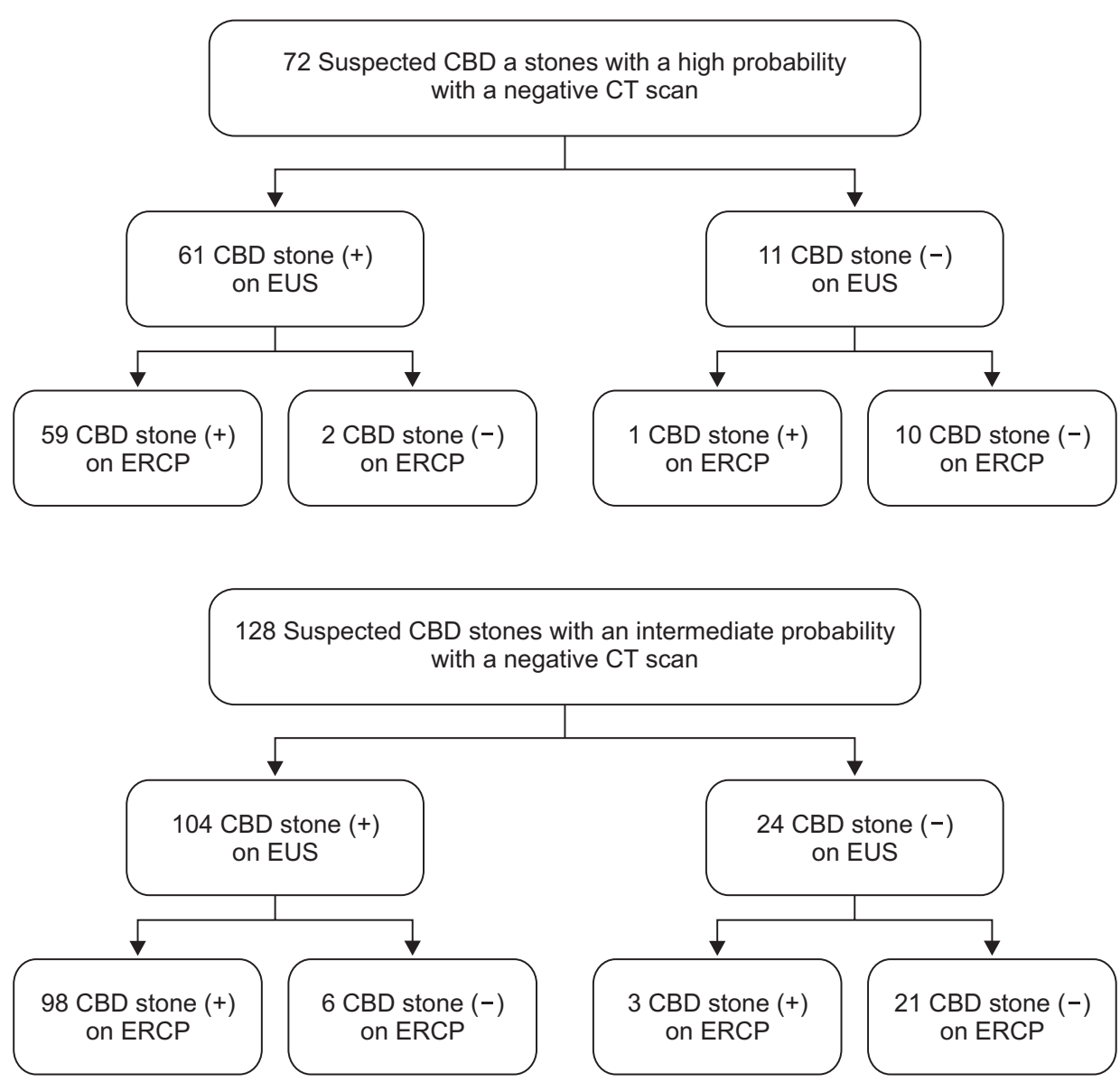

Fig. 3. Suspected common bile duct (CBD) stones with a negative computed tomography scan. Patients with a high probability of choledocholithiasis; 60 of 72 patients (83.3\%) had CBD stones, and endoscopic ultrasonography (EUS) indicated choledocholithiasis in 59 of 60 patients (98.3\%).
Fig. 4. Suspected common bile duct (CBD) stones with a negative computed tomography scan. Patients with an intermediate probability; 101 of 128 patients $(78.9 \%)$ had CBD stones, and endoscopic ultrasonography (EUS) indicated choledocholithiasis in 98 of 101 patients (97\%).
72 patients. EUS revealed choledocholithiasis in 59 patients (98.3\%) (Fig. 3). One hundred one patients (78.9\%) with intermediate probability had CBD stones among 128 patients. EUS revealed choledocholithiasis in 98 patients (97\%) (Fig. 4).

The baseline characteristics of the patients are shown in Table 1. The presence of cholangitis, CBD stones detected on EUS, and CBD diameter measured by EUS were significantly different between the choledocholithiasis and noncholedocholithiasis groups ( $\mathrm{p}<0.001, \mathrm{p}<0.001$, and $\mathrm{p}=0.029$, respectively). The diagnostic accuracy of EUS for choledocholithiasis was 94.0\% (95\% CI, $89.4 \%$ to $97.1 \%$ ) with sensitivity of $97.5 \%$ (95\% CI, 93.4\% to $99.2 \%$ ), specificity of $79.5 \%$ (95\% CI, $63.1 \%$ to $90.1 \%$ ), positive predictive value of 95.2\% (95\% CI, 90.3\% to 97.7\%), and negative predictive value of $88.6 \%$ (95\% CI, $72.3 \%$ to $96.3 \%$ ). The false-positive rate for an EUS diagnosis of choledocholithiasis was $4.8 \%$ (8/165; 95\% CI, 2.3\% to 9.7\%) and the false-negative rate was $11.4 \%$ (4/35; 95\% CI, 3.7\% to $27.7 \%)$.

There were 24 cases (12.0\%) of ERCP-related complications, including 14 cases (7.0\%) of mild PEP, nine cases (4.5\%) of minor bleeding, and one case (0.5\%) of infundibulotomy-related perforation (Table 2). In addition, six cases (15.4\%) of complications occurred in the noncholedocholithiasis group during the ERCP. All patients who developed complications recovered uneventfully, and no procedure-related mortalities occurred.
We performed multivariate analysis to identify the independent predictors of the presence of choledocholithiasis, using the factors that significantly differed between the choledocholithiasis and noncholedocholithiasis groups and factors that are generally useful for the prediction of choledocholithiasis. The multivariate analysis revealed that the independent predictors of choledocholithiasis were the presence of CBD stones during EUS (odds ratio [OR], 284.51; 95\% CI, 50.44 to 1,604.67), age of $>55$ years (OR, 8.46; 95\% CI, 1.65 to 43.29), and a clinical diagnosis of cholangitis (OR, 5.84; 95\% CI, 1.23 to 27.75) (Table 3). However, CBD dilation, liver function abnormalities, and ASGE probability were not significant predictors of choledocholithiasis.

\section{DISCUSSION}

Although CT is not the imaging technique of choice for patients with clinically suspected choledocholithiasis and is not mentioned in the ASGE guidelines, it is commonly used for the differential diagnosis of acute abdomen and for the detection of bile duct stones, especially in the emergency room. ${ }^{1,2}$ Interestingly, helical CT scans have a reported sensitivity of 65\% to $88 \%$ and a specificity of $73 \%$ to $97 \%$ for choledocholithiasis. ${ }^{1,2,18}$ However, the ability to detect bile duct stones by CT depends 
Table 1. Baseline Characteristics of the Enrolled Patients

\begin{tabular}{|c|c|c|c|c|}
\hline Characteristic & $\begin{array}{c}\text { All } \\
(n=200)\end{array}$ & $\begin{array}{l}\text { ERCP stone }(+) \\
\quad(n=161)\end{array}$ & $\begin{array}{l}\text { ERCP stone }(-) \\
\quad(n=39)\end{array}$ & p-value \\
\hline Age, yr & $58.01 \pm 17.78$ & $58.65 \pm 18.17$ & $55.33 \pm 15.98$ & 0.297 \\
\hline Age $>55$ yr & $115(57.5)$ & $96(59.6)$ & $19(48.7)$ & 0.146 \\
\hline Sex, male/female & $94 / 106$ & $74 / 87$ & $20 / 19$ & 0.550 \\
\hline \multicolumn{5}{|l|}{ Symptoms at presentation } \\
\hline Biliary pain & $186(93)$ & $149(92.5)$ & 37 (94.9) & 0.610 \\
\hline Fever & $71(35.5)$ & 61 (37.9) & $10(25.6)$ & 0.152 \\
\hline Jaundice & $76(38.0)$ & $57(35.4)$ & $19(48.7)$ & 0.124 \\
\hline Clinical pancreatitis & $60(30.0)$ & $48(29.8)$ & $12(30.8)$ & 0.907 \\
\hline Clinical cholangitis & $109(54.5)$ & 98 (60.9) & $11(28.2)$ & $<0.001^{*}$ \\
\hline \multicolumn{5}{|l|}{ Chemistry at presentation } \\
\hline Total bilirubin, mg/dL & $2.54 \pm 2.11$ & $2.61 \pm 2.18$ & $2.26 \pm 1.77$ & 0.194 \\
\hline ALP, U/L & $157 \pm 129$ & $162 \pm 140$ & $137 \pm 62$ & 0.274 \\
\hline$\gamma \mathrm{GT}, \mathrm{U} / \mathrm{L}$ & $407 \pm 379$ & $414 \pm 388$ & $379 \pm 342$ & 0.428 \\
\hline AST, IU & $246 \pm 306$ & $252 \pm 313$ & $220 \pm 280$ & 0.516 \\
\hline ALT, IU & $234 \pm 250$ & $229 \pm 235$ & $253 \pm 306$ & 0.377 \\
\hline Total bilirubin $>4 \mathrm{mg} / \mathrm{dL}$ & $158(79.0)$ & 125 (77.6) & 33 (84.6) & 0.233 \\
\hline LFT $>2$ ULN & $151(75.5)$ & $122(75.8)$ & $29(74.4)$ & 0.500 \\
\hline \multicolumn{5}{|l|}{ EUS findings } \\
\hline Gallstone & $122(61.0)$ & $97(60.2)$ & $25(64.1)$ & 0.636 \\
\hline CBD stone & 165 (82.5) & $157(97.5)$ & $8(20.5)$ & $<0.001^{*}$ \\
\hline CBD dilation, $>6 \mathrm{~mm}$ & $133(66.5)$ & $109(67.7)$ & $24(61.5)$ & 0.464 \\
\hline CBD diameter, mm & $4.57 \pm 2.07$ & $4.65 \pm 2.07$ & $3.01 \pm 1.52$ & $0.029 *$ \\
\hline
\end{tabular}

Data are presented as the number (\%) or mean \pm SD.

ERCP, endoscopic retrograde cholangiopancreatography; ALP, alkaline phosphatase; $\gamma \mathrm{GT}, \gamma$-glutamyl transferase; AST, aspartate aminotransferase; ALT, alanine aminotransferase; LFT, liver function test; ULN, upper limit of normal; EUS, endoscopic ultrasonography; CBD, common bile duct. ${ }^{*} \mathrm{p}<0.05$.

Table 2. Adverse Events of Endoscopic Retrograde Cholangiopancreatography

\begin{tabular}{lccc}
\hline \multicolumn{1}{c}{ Adverse event } & $\begin{array}{c}\text { ERCP stone (+) } \\
(\mathrm{n}=161)\end{array}$ & $\begin{array}{c}\text { ERCP stone }(-) \\
(\mathrm{n}=39)\end{array}$ & $\begin{array}{c}\text { Total } \\
(\mathrm{n}=200)\end{array}$ \\
\hline Pancreatitis* $^{*}$ & $9(5.6)$ & $5(12.8)$ & $14(7)$ \\
Bleeding $^{\dagger}$ & $8(5.0)$ & $1(2.6)$ & $9(4.5)$ \\
Perforation $^{\ddagger}$ & $1(0.6)$ & 0 & $1(0.5)$ \\
Hyperamylasemia $^{\S}$ & $12(7.5)$ & $4(10.3)$ & $16(8.0)$ \\
Total & $30(18.6)$ & $10(25.6)$ & $40(20)$ \\
\hline
\end{tabular}

Data are presented as the number (\%).

ERCP, endoscopic retrograde cholangiopancreatography.

${ }^{*}$ All cases were mild post-ERCP pancreatitis; ${ }^{\dagger}$ All cases were minor bleeding; ${ }^{\ddagger}$ Perforation occurred during infundibulotomy; ${ }^{5}$ Post-ERCP hyperamylasemia is defined as a 3-fold or greater increase in the serum amylase level 24 hours after ERCP.

on a number of stone-related factors (size, shape, position, and density), bile duct diameter (dilated vs nondilated), CT technology (conventional vs helical), CT technique (slice thickness, reconstruction interval, pitch, $\mathrm{kVp}$, and administration of con- trast material), patient selection, and interpreter variability. ${ }^{2,18}$ Among these factors, the small size of bile duct stones and isoattenuation can make them more difficult to identify, ${ }^{2,6}$ and cholesterol stones are iso- or slightly hypo-attenuated (relative to bile), which makes them especially difficult to detect. ${ }^{2,18}$ In this study, 92 patients (57.1\%) had a cholesterol stone, and 79 (49.1\%) had a stone with a diameter of $<4 \mathrm{~mm}$. All patients in this study underwent ultrasonography to measure bile duct diameter, as mentioned in ASGE guidelines. However, a limitation of ultrasonography to evaluate full extrahepatic bile duct is that it could easily be disturbed by stomach gas or an obese abdomen.

ERCP is highly accurate for diagnosing bile duct stones, even if the stones are not detected via CT, and it has been the gold standard for preoperative visualization of the bile duct for many years. ${ }^{1,9,19,20}$ However, the nonselective use of ERCP in patients with suspected choledocholithiasis only detects CBD stones in $<50 \%$ of cases, and $>50 \%$ of patients undergo an unnecessary invasive procedure, with its associated risk of morbidity and mortality. $2,6,8,9$ 
Table 3. Results of the Multivariate Analysis of the Predictors of Choledocholithiasis

\begin{tabular}{lcc}
\hline \multicolumn{1}{c}{ Variable } & OR (95\% CI) & p-value \\
\hline Age $>55$ yr & $8.46(1.65-43.29)$ & $0.010^{*}$ \\
Clinical cholangitis & $5.84(1.23-27.75)$ & $0.026^{*}$ \\
CBD stone (+) on EUS & $284.51(50.44-1604.67)$ & $<0.001^{*}$ \\
CBD diameter $>6$ mm on EUS & $3.02(0.74-12.34)$ & 0.125 \\
LFT $>$ 2 ULN & $1.28(0.25-6.46)$ & 0.768 \\
ASGE (high vs intermediate probability) & $1.85(0.39-8.75)$ & 0.437 \\
\hline
\end{tabular}

OR, odds ratio; CI, confidence interval; CBD, common bile duct; EUS, endoscopic ultrasonography; LFT, liver function test; ULN, upper limit of normal.

${ }^{*} \mathrm{p}<0.05$.

In the last decade, many studies have demonstrated that EUS is as accurate as ERCP for detecting CBD stones, and that it has lower mortality and morbidity rates. Furthermore, diagnostic EUS is rarely associated with complications, compared to complication rates of $5 \%$ to $6 \%$ and mortality rates of $0.07 \%$ to $0.1 \%$ after diagnostic ERCP. ${ }^{3,6,21-25}$ In the present study, we did not observe any complications that were associated with EUS, although ERCP was associated with 24 cases (12\%) of complications (excluding hyperamylasemia). Although a simple comparison of the complication frequencies for EUS and ERCP did not reveal a significant difference, six patients (15.4\%) in the noncholedocholithiasis group experienced ERCP-related complications, such as PEP and bleeding.

In previous studies of EUS for detecting CBD stones, all subjects were usually cholangitis patients. Moreover, there is a lack of research regarding the necessity of immediate ERCP in patients with an intermediate or high probability of having a CBD stone (based on the ASGE criteria) and negative imaging findings. Therefore, we focused on this group when evaluating EUS as the initial approach to CBD stone identification.

EUS results are not influenced by bile duct diameter and stone size. ${ }^{5,20,26}$ In contrast, cholangiography often fails to detect CBD stones that have a diameter of $<4 \mathrm{~mm}$, especially in the dilated CBD. Therefore, because small stones may be mobile and move toward the intrahepatic bile ducts, the operator might fail to detect the stone. In addition, jet injection of contrast media into the $\mathrm{CBD}$ or overfilling of the bile ducts may decrease the resolution of the cholangiogram for stone detection. ${ }^{6}$

Previous studies have suggested that EUS should be used before ERCP in patients with an intermediate probability of choledocholithiasis. ${ }^{1,67,27,28}$ However, the management algorithm for choledocholithiasis (based on the ASGE probability of choledocholithiasis) has several limitations. For example, the criteria for determining the risk of having choledocholithiasis are not well defined, and Buscarini et al..$^{29}$ have reported that choledocholithiasis was present in $<50 \%$ of patients who were classified as being at intermediate risk of having biliary obstruction (based on their clinical, biochemical, and sonographic findings). In addition, liver function tests (including total bilirubin) are commonly used for risk stratification, although they may be affected by other diseases (e.g., systemic sepsis or chronic liver disease). ${ }^{9}$ Furthermore, according to the ASGE guidelines, a total bilirubin of $>4 \mathrm{mg} / \mathrm{dL}$ is a very strong predictor of choledocholithiasis, and direct ERCP is recommended. ${ }^{4}$ However, our results indicated that an elevated total bilirubin level was not a significant predictor of choledocholithiasis in our multivariate analysis. Furthermore, CBD dilation, clinical gallstone pancreatitis, and ASGE probability did not significantly differ between our study groups. Moreover, among the ASGE factors that are very strong or strong-to-moderate predictors, only the presence of clinical cholangitis and age of $>55$ years were strong predictors of choledocholithiasis in the present study; the presence of CBD stones during EUS was also a strong predictor. In the present study, the ASGE criteria provided a diagnostic rate for CBD stones of $83.0 \%$ in the high probability group and $78.9 \%$ in the intermediate group. Therefore, these criteria provided a less accurate diagnosis, compared to the use of EUS. Although ERCP was recommended for patients in the high probability group, our data showed that 12 patients (16.7\%) among 72 patients had no choledocholithiasis according to ERCP whereas EUS revealed that 10 (83.3\%) out of 12 patients. Furthermore, if choledocholithiasis was detected on EUS, the operator could usually perform ERCP immediately. Thus, we suggest a revision of the ASGE criteria to recommend that EUS should be used to select patients for therapeutic ERCP and to avoid diagnostic ERCP in patients with intermediate or high probability of CBD stone with negative CT findings.

On the other hand, because "CBD stone(+) on EUS" may affect the results of multivariate analysis, we also performed the multivariate analysis after excluding the EUS findings. Only cholangitis showed a significant predictor for predicting the presence of choledocholithiasis $(p=0.01)$ and age more than 55 was not a predictor $(p=0.475)$. However, because the $p$-value of ASGE criterion for high versus intermediate probability was 0.06 , the ASGE criterion should not be overlooked.

There are several limitations that warrant careful interpretation of our findings. First, this was a retrospective analysis, which has inherent design-related limitations such as minor 
selection bias. Second, the specificity of EUS for choledocholithiasis was lower than the specificity that was reported in previous studies. Third, although EUS can clearly identify the distal CBD, it can fail to identify the far distal CBD, ampulla of Vater, and perihilar bile duct. Nevertheless, we considered that stones in these areas might not be identified by EUS, and so we paid careful attention to these areas. Fourth, ERCP as a confirmatory criterion standard might miss small stones. In this study, ERCP failed to confirm the presence of CBD stones in eight patients with positive EUS findings. This discrepancy may be related to this limitation or spontaneous stone passage.

In the present study, we evaluated the usefulness of EUS in patients with intermediate or high probability of CBD stones and negative CT findings. Given the limited sensitivity of CT, the risks of ERCP-related complications, and the high diagnostic accuracy and safety of EUS, we propose that EUS may be a suitable method in these groups when determining whether patients should undergo more invasive ERCP procedures.

\section{CONFLICTS OF INTEREST}

No potential conflict of interest relevant to this article was reported.

\section{ACKNOWLEDGEMENTS}

This research was supported by the Gachon University Gil Medical Center (Jae Hee Cho, Grant number: 2013-49), Basic Science Research Program (Jae Hee Cho) through the National Research Foundation of Korea (NRF) funded by the Ministry of Education, Science and Technology (number: 2011-0013944).

\section{REFERENCES}

1. Kim KM, Lee JK, Bahng S, et al. Role of endoscopic ultrasonography in patients with intermediate probability of choledocholithiasis but a negative CT scan. J Clin Gastroenterol 2013;47:449-456.

2. Bang BW, Hong JT, Choi YC, et al. Is endoscopic ultrasound needed as an add-on test for gallstone diseases without choledocholithiasis on multidetector computed tomography? Dig Dis Sci 2012;57:3246-3251.

3. De Lisi S, Leandro G, Buscarini E. Endoscopic ultrasonography versus endoscopic retrograde cholangiopancreatography in acute biliary pancreatitis: a systematic review. Eur J Gastroenterol Hepatol 2011;23:367-374.

4. ASGE Standards of Practice Committee, Maple JT, Ben-Menachem $\mathrm{T}$, et al. The role of endoscopy in the evaluation of suspected choledocholithiasis. Gastrointest Endosc 2010;71:1-9.

5. Ney MV, Maluf-Filho F, Sakai P, Zilberstein B, Gama-Rodrigues J, Rosa H. Echo-endoscopy versus endoscopic retrograde cholangiography for the diagnosis of choledocholithiasis: the influence of the size of the stone and diameter of the common bile duct. Arq
Gastroenterol 2005;42:239-243.

6. Karakan T, Cindoruk M, Alagozlu H, Ergun M, Dumlu S, Unal S. EUS versus endoscopic retrograde cholangiography for patients with intermediate probability of bile duct stones: a prospective randomized trial. Gastrointest Endosc 2009;69:244-252.

7. Polkowski M, Regula J, Tilszer A, Butruk E. Endoscopic ultrasound versus endoscopic retrograde cholangiography for patients with intermediate probability of bile duct stones: a randomized trial comparing two management strategies. Endoscopy 2007;39:296303.

8. Petrov MS, Savides TJ. Systematic review of endoscopic ultrasonography versus endoscopic retrograde cholangiopancreatography for suspected choledocholithiasis. Br J Surg 2009;96:967-974.

9. Lee YT, Chan FK, Leung WK, et al. Comparison of EUS and ERCP in the investigation with suspected biliary obstruction caused by choledocholithiasis: a randomized study. Gastrointest Endosc 2008;67:660-668.

10. Ainsworth AP, Rafaelsen SR, Wamberg PA, Durup J, Pless TK, Mortensen MB. Is there a difference in diagnostic accuracy and clinical impact between endoscopic ultrasonography and magnetic resonance cholangiopancreatography? Endoscopy 2003;35:10291032.

11. Scheiman JM, Carlos RC, Barnett JL, et al. Can endoscopic ultrasound or magnetic resonance cholangiopancreatography replace ERCP in patients with suspected biliary disease? A prospective trial and cost analysis. Am J Gastroenterol 2001;96:2900-2904.

12. Materne R, Van Beers BE, Gigot JF, et al. Extrahepatic biliary obstruction: magnetic resonance imaging compared with endoscopic ultrasonography. Endoscopy 2000;32:3-9.

13. Kondo S, Isayama $\mathrm{H}$, Akahane $\mathrm{M}$, et al. Detection of common bile duct stones: comparison between endoscopic ultrasonography, magnetic resonance cholangiography, and helical-computedtomographic cholangiography. Eur J Radiol 2005;54:271-275.

14. de Lédinghen V, Lecesne R, Raymond JM, et al. Diagnosis of choledocholithiasis: EUS or magnetic resonance cholangiography? A prospective controlled study. Gastrointest Endosc 1999;49:26-31.

15. Verma D, Kapadia A, Eisen GM, Adler DG. EUS vs MRCP for detection of choledocholithiasis. Gastrointest Endosc 2006;64:248254.

16. Takada T, Strasberg SM, Solomkin JS, et al. TG13: updated Tokyo Guidelines for the management of acute cholangitis and cholecystitis. J Hepatobiliary Pancreat Sci 2013;20:1-7.

17. Banks PA, Bollen TL, Dervenis C, et al. Classification of acute pancreatitis--2012: revision of the Atlanta classification and definitions by international consensus. Gut 2013;62:102-111.

18. Anderson SW, Lucey BC, Varghese JC, Soto JA. Accuracy of MDCT in the diagnosis of choledocholithiasis. AJR Am J Roentgenol 2006;187:174-180.

19. Napoléon B, Dumortier J, Keriven-Souquet O, Pujol B, Ponchon T, Souquet JC. Do normal findings at biliary endoscopic ultrasonography obviate the need for endoscopic retrograde cholangiography in patients with suspicion of common bile duct stone? A prospec- 
tive follow-up study of 238 patients. Endoscopy 2003;35:411-415.

20. Kohut M, Nowak A, Nowakowska-Dulawa E, Marek T, Kaczor

R. Endosonography with linear array instead of endoscopic retrograde cholangiography as the diagnostic tool in patients with moderate suspicion of common bile duct stones. World J Gastroenterol 2003;9:612-614.

21. Tse F, Liu L, Barkun AN, Armstrong D, Moayyedi P. EUS: a metaanalysis of test performance in suspected choledocholithiasis. Gastrointest Endosc 2008;67:235-244.

22. Aljebreen A, Azzam N, Eloubeidi MA. Prospective study of endoscopic ultrasound performance in suspected choledocholithiasis. J Gastroenterol Hepatol 2008;23:741-745.

23. Garrow D, Miller S, Sinha D, et al. Endoscopic ultrasound: a metaanalysis of test performance in suspected biliary obstruction. Clin Gastroenterol Hepatol 2007;5:616-623.

24. Meroni E, Bisagni P, Bona S, et al. Pre-operative endoscopic ultrasonography can optimise the management of patients undergoing laparoscopic cholecystectomy with abnormal liver function tests as the sole risk factor for choledocholithiasis: a prospective study.
Dig Liver Dis 2004;36:73-77.

25. Prat F, Amouyal G, Amouyal P, et al. Prospective controlled study of endoscopic ultrasonography and endoscopic retrograde cholangiography in patients with suspected common-bileduct lithiasis. Lancet 1996;347:75-79.

26. Liu CL, Fan ST, Lo CM, et al. Comparison of early endoscopic ultrasonography and endoscopic retrograde cholangiopancreatography in the management of acute biliary pancreatitis: a prospective randomized study. Clin Gastroenterol Hepatol 2005;3:1238-1244.

27. Janssen J, Halboos A, Greiner L. EUS accurately predicts the need for therapeutic ERCP in patients with a low probability of biliary obstruction. Gastrointest Endosc 2008;68:470-476.

28. Sgouros SN, Bergele C. Endoscopic ultrasonography versus other diagnostic modalities in the diagnosis of choledocholithiasis. Dig Dis Sci 2006;51:2280-2286.

29. Buscarini E, Tansini P, Vallisa D, Zambelli A, Buscarini L. EUS for suspected choledocholithiasis: do benefits outweigh costs? A prospective, controlled study. Gastrointest Endosc 2003;57:510-518. 